\title{
Retos y aprendizajes para el manejo de eventos extremos por cambio climático. Caso de estudio: el sistema de alerta temprana comunitaria en la cuenca del río Bogotá (Colombia) como medida de adaptación ante la amenaza de inundación
}

Challenges and lessons learned for managing extreme events due to climate change. Case study: the community early warning system in the Bogotá river (Colombia) basin as an adaptation measure to the threat of flooding

PALACIO GOMEZ, Diana C. ${ }^{1}$

\section{Resumen:}

En este artículo se discute sobre la posibilidad e importancia de implementar un SAT Comunitario en la cuenca del río Bogotá como medida de adaptación ante el posible aumento de la amenaza de inundación por comportamientos no estacionarios de procesos meteorológicos e hidrológicos debido al cambio climático.

Palabras claves: sistema de alerta temprana comunitaria, amenaza, inundación, cambio climático, no estacionario.

\begin{abstract}
:
This paper discusses the possibility and importance of implementing a Community SAT in the Bogotá river basin as a measure of adaptation to the possible increase in the threat of flooding due to nonstationary behavior of meteorological and hydrological processes due to climate change.

Keywords: community early warning system, threat, flooding, climate change, not stationary.
\end{abstract}

\section{Introducción}

En el espacio geográfico de la cuenca del río Bogotá se concentra la mayor densidad de población por kilómetro cuadrado del país, específicamente en la ciudad de Bogotá, capital de la nación y en donde se ha construido la mayor cantidad de viviendas del mismo, con una concentración de gran diversidad de culturas, creencias y costumbres, dado que en la capital del país conviven personas de muchas regiones del país.

De otra parte, las proyecciones del Instituto de Hidrología, Meteorología y Estudios Ambientales (IDEAM), en la 2a Comunicación Nacional ante la Convención Marco de las Naciones Unidas sobre Cambio Climático en 2010, la

\footnotetext{
${ }^{1}$ Ingeniera en Recursos Hídricos y Gestión Ambiental, Especialista, Master en Gestión del Riesgo. Docente del programa de Ingeniería Civil De la Escuela Militar de Cadetes “General José María Córdova”. Email: diana.palacio@esmic.edu.co
} 
Corporación Autónoma Regional de Cundinamarca (CAR) en el informe del autor Pabón Caicedo en $2011^{2}$ y del Distrito Capital de Bogotá en su proyecto concerniente a gestión integral del riesgo en los componentes de pronóstico hidrológico y alerta temprana en la cuenca del río Bogotá en 2012 y las consideraciones metodológicas del Panel Intergubernamental del Cambio Climático (IPCC) de la Organización Meteorológica Mundial (OMM), aseveran que bajo condiciones futuras de los escenarios ante cambio climático, se presentarán transformaciones drásticas en el comportamiento de la atmósfera e hidrósfera en la cuenca del río Bogotá. Ello traería como consecuencia una alteración radical en la frecuencia de las inundaciones con las consecuencias respectivas en la infraestructura de transporte, viviendas, agua potable y saneamiento.

Es así como se presenta una propuesta de sistema de alerta temprana con énfasis en el esfuerzo comunitario de las gentes, con soporte en el modelo de tipo no estacionario. Este tipo de sistema es poco conocido en Colombia, toda vez que las emisiones de pronósticos del comportamiento de los procesos en la atmo-hidrósfera y sus consecuentes alertas se imparten exclusivamente con soporte en los aspectos científicos y no tienen en cuenta su asimilación por parte de las personas, ni tampoco el aporte que éstas puedan realizar para mejorar los resultados de las alertas ante los eventos de inundación.

\subsection{Reseña}

Desde el ámbito de la hidrología, los eventos de inundación son procesos físicos que permiten transformar energía del agua y que tienen como consecuencia el desborde de las aguas de un río de manera súbita (en los ríos de montaña) o pausada (en los ríos de planicie). En ellos, intervienes diversos procesos que se suceden en la atmósfera, tales como la radiación solar, la evaporación, la precipitación, la temperatura del aire, entre otros, que se relación en el concepto hidrológico de Balance Hídrico: cuando las lluvias exceden la capacidad de asimilación en el cauce de un río, las aguas se sobresalen de los cauces con las respectivas consecuencias. En hidrología se sabe que las inundaciones son naturales y obedecen como eventos a leyes físicas que relacionan diversos procesos presentes en la atmósfera y en la hidrósfera.

Desde el punto de vista de las personas que habitan en la cuenca del río Bogotá, los eventos de inundación se suelen interpretar de dos maneras: a) algunos consideran que las inundaciones son hechos que traen grandes beneficios y bendiciones, puesto que al desbordarse el agua de los cauces, no sólo se esparcen las aguas por las orillas de los ríos sino también llegan nuevas cantidades de minerales y sustancias que enriquecen sus tierras para los posteriores cultivos; b) otros ven en las inundaciones sólo pérdidas, dado que se inundan sus viviendas, se pierden los cultivos, se destruyen las vías terrestres, etc.

En la cuenca del río Bogotá, en la cual se encuentran geográficamente ubicados más de 10 municipios del departamento de Cundinamarca, se tienen diversas reseñas sobre eventos de inundación desde inicios del siglo $X X$. Los expertos en hidrología confirman que las inundaciones con mayores incidencias en lo económico y social se presentaron durante la ola invernal de los años 2010-2011, conocida en el ámbito ambiental como Fenómeno de la NIÑA.

De acuerdo con el informe reporte final de áreas afectadas por inundaciones (2010 -2011) elaborado por el Instituto Geográfico Agustín Codazzi (IGAC), Departamento Nacional de Planeación (DANE) y el Instituto de

2 Pabón Caicedo, J. D. (2011). El cambio climático en el territorio de la Corporación Autónoma Regional de Cundinamarca. 
Hidrología, Meteorología y Estudios Ambientales (IDEAM), en junio de 2011, se muestra la estadística de afectaciones de la emergencia invernal de los años 2010-2011 a nivel nacional (ver la tabla 1).

Tabla 1

Relación de las afectaciones en la emergencia invernal de 2010-2011

\begin{tabular}{|l|r|}
\hline \multicolumn{1}{|c|}{ Total 998 Municipios } & \multicolumn{1}{|c|}{ Hogares } \\
\hline Número de hogares con pérdidas agropecuarias & 603.895 \\
\hline Número de hogares con pérdidas de cultivos & 483.929 \\
\hline Número de hogares con pérdida de ganado & 242.137 \\
\hline Número de hogares con pérdida de aves de corral & 263.726 \\
\hline Número de hogares con pérdida de otras especies menores & 46.461 \\
\hline Número de hogares con pérdida de cultivo de peces & 12.715 \\
\hline Número de hogares con afectación de vivienda & 557.377 \\
\hline Número de hogares con afectación en finca o parcela & 300.618 \\
\hline
\end{tabular}

Fuente: IGAC, DANE, IDEAM (2011), reporte final de áreas afectadas por inundaciones 2010 - 2011, recuperado de:

https://www.dane.gov.co/index.php/139-espanol/noticias/ultimasnoticias/1305-reporte-final-de-areas-afectadas-por-inundaciones-2010-2011

Además, de las afectaciones diferentes que se estimaron para cerca del $40 \%$ del país estudiado, es interesante resaltar que muchas zonas inundadas resultaron coincidir con las áreas urbanas en varios departamentos (ver la tabla 2), lo cual nos indica que la ocupación del territorio en el país se ha venido dando en espacios geográficos propios de los ríos.

Tabla 2

Áreas urbanas en zonas de inundación en la emergencia invernal de 2010-2011

\begin{tabular}{|l|r|r|r|r|r|}
\hline Departamento & $\begin{array}{c}\text { Área Urbana } \\
\text { Total (Ha) }\end{array}$ & $\begin{array}{c}\text { Interpretado } \\
\text { del } \\
\text { Departamento }\end{array}$ & $\begin{array}{c}\text { Área } \\
\text { Inundación } \\
\text { Total (Ha) }\end{array}$ & $\begin{array}{c}\text { Área Urbana } \\
\text { en Zona de } \\
\text { Inundación } \\
\text { (Ha) }\end{array}$ & $\begin{array}{c}\text { Porcentaje } \\
\text { del Área } \\
\text { Urbana en } \\
\text { Zona de } \\
\text { Inundación }\end{array}$ \\
\hline META & 13.396 & 25,8 & 96.899 & 486 & 0,502 \\
\hline NARIÑO & 11.157 & 4,1 & 17 & 0 & 0 \\
\hline NORTE DE SANTANDER & 13.180 & 92,4 & 26.403 & 141 & 0,534 \\
\hline QUINDÍO & 4.718 & 70 & 176 & 2 & 0,939 \\
\hline RISARALDA & 6.841 & 97,4 & 1.711 & 2 & 0,135 \\
\hline SANTANDER & 18.628 & 90,5 & 99.964 & 564 & 0,564 \\
\hline SUCRE & 10.545 & 76,7 & 97.940 & 906 & 0,925 \\
\hline TOLIMA & 17.916 & 93 & 13.118 & 185 & 1,41 \\
\hline VALLE DEL CAUCA & 40.514 & 99,1 & 12.176 & 177 & 1,457 \\
\hline Total general & $\mathbf{3 9 7 . 5 3 7}$ & & $\mathbf{1 . 6 4 2 . 1 0 8}$ & $\mathbf{1 7 . 0 9 4}$ & \\
\hline
\end{tabular}

Fuente: IGAC, DANE, IDEAM (2011), reporte final de áreas afectadas por inundaciones 2010 - 2011, recuperado de: https://www.dane.gov.co/index.php/139espanol/noticias/ultimas-noticias/1305-reporte-final-de-areas-afectadas-porinundaciones-2010-2011 
Con la información presentada en las tablas 1 y 2 se puede afirmar que a la fecha del reporte (agosto de 2011), las personas se vieron afectadas no tanto por los mismos eventos de inundación sino más bien por haber impuesto una ocupación del territorio en el país no adecuado: las zonas en donde se explayan las aguas de los ríos, lagos, ciénagas y otros cuerpos hídricos, son espacios geográficos que ameritan especial atención para ser ocupados, so pena de sufrir las consecuencias vividas en la emergencia invernal de 2010-2011.

Al respecto se afirma que las afectaciones de las inundaciones en el país son consecuencias de haber juntado tantas personas en un espacio geográfico relativamente pequeño y ello se debe principalmente a los siguientes factores: a) ausencia de planeación para vivir en estos espacios; b) ubicación de la población en sitios con insuficiente cantidad de agua para su abastecimiento; c) ubicación de las gentes en sitios proclives a eventos de sismos; d) ubicación de viviendas y fábricas en sitios propensos a eventos de inundación y deslizamientos, entre muchos otros.

Según la Universidad Externado (2007) “como ha sucedido en muchos países desarrollados y con mayor velocidad en la mayoría de países menos desarrollados, en Colombia el proceso de urbanización ha sido muy acelerado. La proporción de población residente en cabeceras municipales del país se multiplicó por doce al pasar de dos millones y medio en 1938 a 31,5 millones en 2005" (p. 13). Así las cosas, nuestro país ha tomado el rumbo de la aglomeración de las gentes en pequeños espacios. Allí mismo encontramos que "la población residente en las cabeceras municipales creció desde 1938 a un ritmo promedio anual de 3.8\% mientras que la población residente fuera de la cabecera, conocida como "resto del municipio", creció a menos de $1 \%$ anual. El período intercensal de mayor crecimiento de población urbana fue el de 1951-1964 (tasa media anual de crecimiento de 5.6\%). Este período también muestra el mayor crecimiento de la población rural, pero con una tasa sensiblemente menor (1.3\%)" (p.13).

Se advierte que "las diferencias en los indicadores de densidad poblacional (habitantes por kilómetro cuadrado en el 2005) muestran una extensa variedad de densidades a lo largo de las cadenas montañosas que definen la geografía nacional (mapa 2). Los municipios con altas densidades de población urbana reproducen la estructura de las tres cordilleras, mostrando que las características geográficas que históricamente marcaron el proceso de colonización y el desarrollo urbano-regional, todavía continúan definiendo regiones bien diferenciadas. Las mayores densidades corresponden a las tierras altas de climas templados o fríos, mientras los valles cálidos interandinos y los llanos y selvas de la Amazonía y la Orinoquía aún tienen grandes extensiones de territorio despoblado. La Costa Caribe que históricamente fuera la puerta de entrada al continente continúa manteniendo altas densidades de población" (Universidad Externado, 2007, p. 17)

Para el caso específico del departamento de Cundinamarca, en el cual se encuentra ubicada espacialmente la cuenca del río Bogotá, se tiene que en materia de daños en bienes, infraestructura y viviendas, aunque no fueron los mayores del país, si fueron significativos (ver tabla 3).

Se puede afirmar que, a pesar de no haber sufrido los mayores daños en comparación con todo el país, el departamento de Cundinamarca sufrió daños por el valor de unos 500 mil millones de pesos aproximadamente, según el informe de valoración de daños y pérdidas: Ola invernal en Colombia 2010-2011, elaborado por el Banco Interamericano de Desarrollo (BID) y la Comisión Económica para América Latina y el Caribe (CEPAL) en 2012. Es importante resaltar que a la fecha actual ninguna entidad o agencia nacional o internacional ha estudiado o reportado sobre aquellas pérdidas que se hubiesen podido evitar actuado de manera preventiva ante el conocimiento preliminar que se tenía sobre las posibilidades del aumento de las lluvias y sus consecuentes eventos de inundación, ni tampoco sobre las pérdidas posiblemente evitadas si se hubiesen implementado las mejoras en la producción del conocimiento respecto a la gestión del Riesgo. 
Tabla 3

Relación de daños por departamento

(en millones de pesos)

\begin{tabular}{|c|c|c|c|c|c|}
\hline Depto. & Hábitat & $\begin{array}{l}\text { Infraes- } \\
\text { tructura }\end{array}$ & $\begin{array}{c}\text { Servicios sociales } \\
\text { y } \\
\text { administración } \\
\text { pública }\end{array}$ & Productivos & Total \\
\hline Amazonas & 2083 & 2301 & 3249 & 0 & 7633 \\
\hline Antioquia & 227491 & 100970 & 329634 & 41301 & 699396 \\
\hline Arauca & 4871 & 16 & 28498 & 2 & 33387 \\
\hline Atlántico & 650769 & 98802 & 141565 & 15945 & 907081 \\
\hline Bogotá & 16455 & 0 & 79 & 20 & 16554 \\
\hline Bolívar & 631088 & 223399 & 120287 & 203530 & 1178304 \\
\hline Boyacá & 93978 & 11592 & 208208 & 4240 & 318019 \\
\hline Caldas & 145782 & 19198 & 104845 & 4393 & 274219 \\
\hline Caquetá & 32854 & 0 & 27596 & 1135 & 61585 \\
\hline Casanare & 30306 & 0 & 24418 & 0 & 54724 \\
\hline Cauca & 256244 & 21400 & 167492 & 3731 & 448867 \\
\hline Cesar & 197108 & 6279 & 135051 & 43333 & 381771 \\
\hline Chocó & 131855 & 82592 & 28005 & 0 & 242452 \\
\hline Córdoba & 256234 & 71376 & 94512 & 82891 & 505013 \\
\hline Cundinamarca & 89443 & 25261 & 379216 & 32610 & 526530 \\
\hline
\end{tabular}

Fuente: Banco Interamericano de Desarrollo y Comisión Económica para América Latina y el Caribe, (2012). Valoración de daños y pérdidas ola invernal en Colombia 2010-2011

De otra parte, el país ha sido advertido en diversos estudios e informes IDEAM de 2001, 2010, 2013, 2015 y 2018 sobre los posibles impactos que podría ocasionar el fenómeno de cambio climático en materia de los procesos meteorológicos e hidrológicos.

El fenómeno de cambio climático consiste físicamente en el cambio del comportamiento de los procesos que suceden en la atmósfera, tales como la humedad relativa, la temperatura del aire, la precipitación, entre otros. Para estudiar este fenómeno el científico apela al método estadístico y es por ello que el clima, según la OMM (1992) es "la síntesis de las condiciones meteorológicas en un lugar determinado, caracterizada por estadísticas a largo plazo (valores medios, varianzas, probabilidades de valores extremos, etc.) de los elementos meteorológicos en dicho lugar"(p. 113), mientras que el cambio climático "abarca todas las formas de inconstancia climática (esto es, cualesquiera diferencias entre las estadísticas a largo plazo de los elementos meteorológicos calculados para distintos períodos pero respecto a la misma zona" (p. 113). Así las cosas, las proyecciones a futuro del comportamiento de la atmósfera para el caso de Colombia, que realiza el Instituto IDEAM, estadísticamente demuestran que habrá cambio del comportamiento histórico de la atmósfera en el país en los periodos temporales de 2011-2040, 2041-2070 y 2071-2100. Según IDEAM en las Segunda Comunicación Nacional (2010) se tendrán en el territorio nacional aumentos de temperaturas del aire en la zona andina (en donde se ubica la mayor cantidad de áreas urbanas), disminuciones de la humedad relativa del aire y en algunos sitios entre aumentos y disminuciones se debatirán las precipitaciones. Otra conclusión y quizá la más importante, trata sobre el aumento imparable de la frecuencia de los eventos de sequía e inundación en todo el país. 
De acuerdo con los expertos del IDEAM en la Primera Comunicación Nacional de 2001 en Colombia se presentarán diversos efectos del cambio climático en los caudales de los ríos con alteraciones de disminución (menor al 30\% con respecto a la escorrentía histórica) en la gran mayoría de las regiones, sobre todo en la región andina, en la cual se encuentra la cuenca del río Bogotá. De acuerdo a las previsiones de los posibles impactos del cambio climático por calentamiento, se debe considerar que los eventos de inundación podrían llegar a ser más frecuentes y con mayores desbordamientos de las aguas en los ríos y demás cuerpos hídricos.

De acuerdo con las experiencias foráneas en el tema de cambio climático, existen alternativas científicas para afrontar sus efectos con garantía de éxito, en el sentido de aminorar sus consecuencias. Una de estas alternativas trata sobre las medidas de adaptación, las cuales son decisiones temporales o parciales que se toman ante escenarios de riesgo, abordadas desde una óptica integradora que incluye los escenarios de riesgo como tal, el análisis de los grupos de interés involucrados o tomadores de decisiones, el análisis costo-beneficio y los árboles de decisión. (Aldunce P. et al, 2008 p.44).

De acuerdo con Aldunce y otros (2008), la adaptación corresponde a "un ajuste de los sistemas humanos a cambios de las condiciones climáticas, con el objeto de reducir la vulnerabilidad. La adaptación resulta de un proceso de toma de decisiones y representa una oportunidad de mejorar a través de transformaciones en tecnología, educación, comportamiento, política pública, o infraestructura, es un proceso de transformación flexible pero no la decisión definitiva" (p. 44). De acuerdo con el Grupo Intergubernamental de Expertos sobre el Cambio Climático, la adaptación puede ser categorizada en planeada o autónoma (Agard, J., Schipper, E. L, Birkmann, J., Campos, M., Dubeux, C., Nojiri, Y., \& Mastrandrea, M. D., 2001, p.2).

En los Planes de Ordenamiento Territorial o Esquema de Ordenamiento se deben incluir las medidas de adaptación ante las consecuencias de los impactos del fenómeno de cambio climático y el aumento de los riesgos por los eventos de inundación en las áreas urbanas del país. Para ello, es importante elaborar las estimaciones de la vulnerabilidad presente y futura. En (Aldunce P. et al, 2008) encontramos que "una forma útil y simplificada de pensar en vulnerabilidad es en función de los impactos y la adaptación. Así, los impactos dependen de la exposición del sistema al clima y de su sensibilidad, mientras que la adaptación depende de la capacidad del sistema de reducir su vulnerabilidad y del uso que se haga de la tal capacidad. Por ello, para poder iniciar el proceso de adaptación es necesario generar capacidad adaptativa, es decir, habilidad para ajustar un sistema a condiciones climática tanto presentes como futuras, con el fin de aminorar sus potenciales impactos negativos o para sacar ventaja de los aspectos positivos" (p. 45).

Para culminar esta reseña, debemos señalar que los Sistemas de Alerta Temprana (SAT), en sí son una medida de adaptación adecuada, dado que representan un conjunto de acciones, tecnología, instrumentos y conocimientos que permiten con soporte en el comportamiento actual e histórico, prever la dinámica del agua a futuro y es por ello que logran predecir los alcances espaciales (hasta donde llegarán) y temporales (hasta cuando durarán) de los eventos de inundación. En Colombia no se ha logrado encontrar experiencias municipales en las áreas urbanas que permitan conocer con cierto tiempo de antelación la magnitud cuantitativa de las inundaciones. Los mejores esfuerzos pertenecen al Instituto IDEAM y a la administración de ciudades como Bogotá, Medellín y Cali. Sin embargo, en ninguna de estas entidades se aplica el sentido comunitario en las etapas de monitoreo hidro-meteorológico, pronóstico hidro-meteorológico y emisión de las alertas. Tanto en el IDEAM, como en Medellín, Cali y Bogotá, se aplica exclusivamente conocimiento científico.

Los SAT son tan antiguos como las necesidades humanas de sobrevivir en su época primitiva. Ha existido una gran diversidad de SAT en los ámbitos sociales, económicos y espirituales. Son famosos los mitos, leyendas, cuentos y demás expresiones culturales sobre las señales que existen en la naturaleza humana y divina para 
detectar que en el quehacer diario algo no está bien y que se debe corregir so pena de vivir los castigos correspondientes. En este sentido, es importante resaltar que antes de que existieran las consideraciones científicas para alertar algún comportamiento natural que conllevase un peligro o amenaza al ser humano, existieron saberes humanos que sin rigurosidad científica lograban advertir sobre los hechos adversos. Es así como desde la historia antigua se ha explicado que las gentes comentaban de boca a oído los designios divinos y los hechos que resultarían en beneficio para la vida. Los sacerdotes del antiguo Egipto y los Chamanes en tierras de América, eran los seres humanos que mantenían una comunicación directa con las deidades y transmitían sus voluntades a los demás hombres: cualquier enojo en las divinidades contraería peligros, amenazas, situaciones desagradables y de castigo. Ellos en sus tierras desarrollaron ciertas habilidades que les permitía "ver" el estado actual en las cosas, "prever" lo que podría suceder a corto, mediano y largo plazo y también aconsejaban a los humanos que ostentaban el poder visible para que pudiesen "decidir" o actuar de tal forma que los castigos divinos se evitaran.

La estructura gubernamental actual en Colombia asigna responsabilidades en entidades de índole nacional, regional y local. Ello está establecido en diversas leyes, decretos y resoluciones en cada uno de los ministerios públicos y su máxima expresión en los ámbitos del riesgo se encuentra plasmada en la reciente Ley de Riesgos No. 1523 de 2012. Una de las evidencias de la necesidad latente de unir consideraciones científicas y culturales en materia de los SAT es que en esta ley ya no se habla de "desastres naturales" (como sí lo siguen haciendo en otros países) sino de "desastres" toda vez que los seres humanos han entendido que la naturaleza tiene sus propias dinámicas y los seres humanos deben adaptarse a ellas; ya el hombre no es tan poderoso como lo señalaba en el siglo XVII el científico Isaac Newton.

Hemos visto que los asentamientos humanos en las áreas urbanas del país vienen en aumento, que las políticas de ocupación del territorio no han sido las mejores desde el punto de vista de la planeación, que las inundaciones son eventos naturales con alta frecuencia y que durante la ola invernal de 2010-2011 su impacto afectó cerca de 1.000 municipios en todo el país. A raíz de la madurez social, económica y política, y teniendo como hecho culminante a la emergencia invernal, el gobierno nacional expidió en el año de 2012 la Ley 1523. La ley 1523, por la cual se adopta la política nacional de gestión del riesgo de desastres y se establece el Sistema Nacional de Gestión del Riesgo de Desastres y se dictan otras disposiciones, contempla varias medidas y decisiones para fortalecer la identificación de la vulnerabilidad y la amenaza y agilizar las relaciones entre las instituciones que deben afrontar las situaciones de desastres y riesgos. En esta Ley, por primera vez desde la norma legal se establece el concepto de riesgo como una relación existente entre el daño posible que puede ocasionar un evento natural o antropogénico y la amenaza. Antes de esta Ley, en el país se consideraba que el riesgo era una amenaza y no existía el concepto legal de vulnerabilidad. En este sentido, a partir del año 2012, para conocer el riesgo en un sitio y tiempo determinado se deben estimar la vulnerabilidad y la amenaza. La vulnerabilidad es un concepto que aún no cuenta con las metodologías acordadas entre los expertos que permitan estimar los costos de los posibles daños ante la probabilidad de que se presente un evento adverso (que se conoce como amenaza).

\section{Metodología}

Para desarrollar el presente trabajo se procede a realizar una investigación documental, haciendo un análisis descriptivo de la información bibliográfica encontrada, para lo cual, se consultaron documentos en donde se presentan los métodos y conceptos que se han utilizado en los estudios orientados a eventos por inundaciones y sistemas de alerta temprana -SAT tanto en organismos públicos y privados de Colombia como de organismos internacionales. 


\section{Los SAT : Contexto internacional, nacional y local}

\subsection{El concepto de SAT a nivel internacional}

En la Estrategia Internacional para la Reducción de Desastres encontramos la recomendación de considerar a un SAT como "el conjunto de capacidades necesarias para generar y difundir información de alerta que sea oportuna y significativa, con el fin de permitir que las personas, las comunidades y las organizaciones amenazadas por una amenaza se preparen y actúen de forma apropiada y con suficiente tiempo de anticipación para reducir la posibilidad de que se produzcan pérdidas o danos". (ONU, 2019, p.33).

De acuerdo con la OEA (2010) un SAT "consiste en la transmisión rápida de datos que active mecanismos de alarma en una población previamente organizada y capacitada para reaccionar. El suministro de información oportuna se realiza por medio de las instituciones encargadas, lo que permite a las personas expuestas a la amenaza tomar acciones para reducir el riesgo y prepararse para una respuesta efectiva" (p.39).

Para el Centro de Coordinación para la Prevención de los Desastres Naturales en América Central, CEPREDENAC, un SAT "Comprende la suma de las políticas, estrategias, instrumentos y acciones particulares referidos a la identificación y monitoreo de amenazas, vulnerabilidades y riesgo, el diseño e implementación de alertas o alarma relacionada con la ocurrencia inminente de eventos peligrosos; los preparativos para la respuesta a emergencias y la ejecución de los mismos" (CEPREDENAC, 2007, p.30) tomado de (OEA, 2010, p.3)

En la EIRD se presentan los requisitos mínimos que debería tener un SAT: a) Conocimiento del riesgo; b) Seguimiento de cerca o monitoreo; c) Análisis y pronóstico de las amenazas; d) Comunicación o difusión de las alertas y los avisos; y e) Capacidades locales para responder frente a la alerta recibida. (ONU, 2009, p.12). A nivel internacional se suelen diferenciar dos tipos principales de SAT: a) el que se acciona de manera central, como lo es el caso de Colombia con su estructura gubernamental y científica, en donde la autoridad por norma legal en materia meteorológica e hidrológica le corresponde al IDEAM; b) aquellos SAT accionados por las mismas comunidades en cada una de sus etapas o elementos, mezclando el conocimiento científico con la sabiduría popular.

De acuerdo con el "Manual para el diseño, instalación, operación y mantenimiento de sistemas comunitarios de alerta temprana ante inundaciones", elaborado por expertos de la Organización de los Estados Americanos- OEA y publicado en el año 2010, es importante implementar un Sistema Comunitarios de Alerta Temprana ante Inundaciones - SATIs, debido a la necesidad de tomar acciones que permitan reducir la pérdida de vidas humanas en las comunidades vulnerables ante el desbordamiento de los ríos. El esquema general se ilustra en la figura 1. La implementación de un SATI comprende una serie de etapas que pueden agruparse en dos: (i) una etapa técnica, referida a la instrumentación, medición hidrometeorologica y pronostico; y (ii) una etapa social, referida a la organización comunitaria requerida para garantizar la operación apropiada de los sistemas de alerta ante inundaciones (p.3).

En el Manual para el Diseño e Implementación de un Sistema de Alerta Temprana de Inundaciones en Cuencas Menores, elaborado por expertos de la OEA (2001), encontramos cinco pasos para el diseño y operación de un SAT: a) Organización comunitaria; b) Reconocimiento de la cuenca menor; c) Medición de lluvia y nivel de agua de los ríos; d) Funcionamiento del sistema de alerta; y e) Evaluación de la situación, difusión de la alerta y plan de emergencia. 
Figura 1

Esquema general de un SAT Comunitario

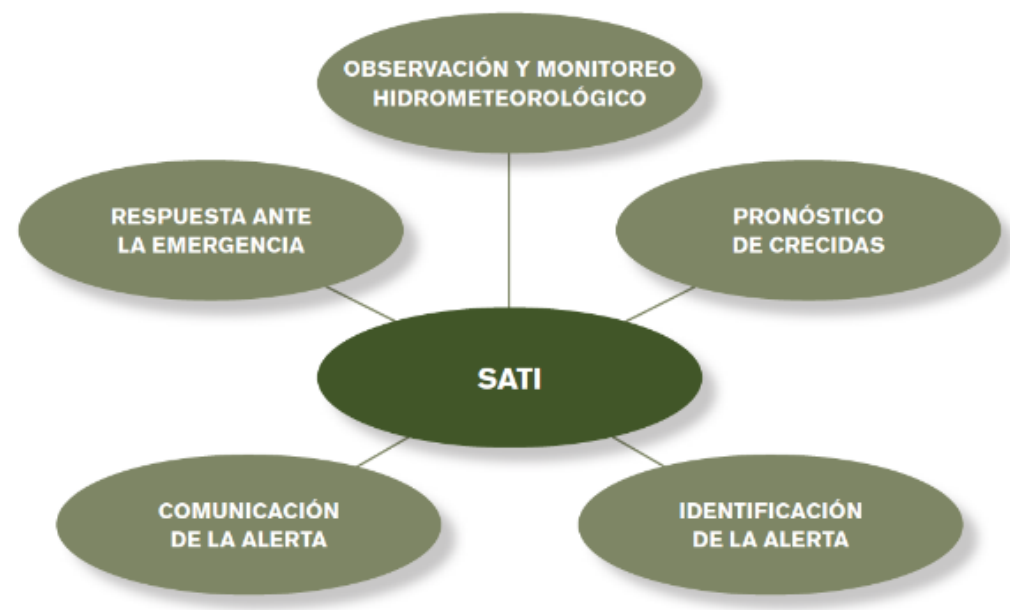

Fuente: OEA, (2010). Manual para el diseño, instalación, operación y mantenimiento de sistemas comunitarios de alerta temprana ante inundaciones

\subsection{El concepto de SAT en Colombia}

Para el caso de Colombia, las etapas que le anteceden a la emisión de una alerta temprana son el monitoreo hidrológico, indicadas en los protocolos para la emisión de los pronósticos hidrológicos de 2007 y 2008 realizados por el IDEAM. En Colombia tanto la alerta como sus etapas antecedentes se llevan a cabo sin tener en cuenta el conocimiento popular de las gentes. La situación de rechazo de la sabiduría popular en las entidades nacionales y locales encargadas de prever los eventos de inundación y sus efectos, aminora significativamente el efecto de un pronóstico de un evento de inundación. El esquema general que describe estas etapas se ilustra en la figura 2.

Figura 2

Toma de decisiones son soporte en un pronóstico hidrológico de inundación

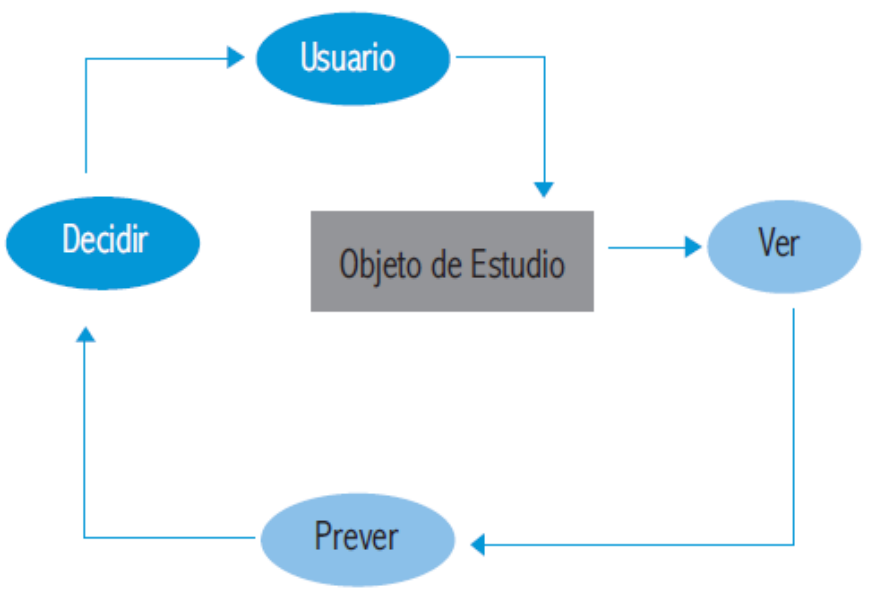

Fuente: IDEAM, (2008), Protocolo para la emisión de los pronósticos hidrológicos. Subdirección de Hidrología. Bogotá. 
De acuerdo al IDEAM (2008), "el funcionamiento operacional de la emisión de un aviso, boletín o propuesta de alerta consiste en lo siguiente: generalmente el objeto de estudio o característica hidrológica es el nivel del agua en determinado sitio, cuya dinámica es observada y medida en tiempo real mediante la red automática de estaciones hidrométricas" (p.22).

Valiéndose de medios estratégicos como la radio y el teléfono también se logran transmitir los datos de niveles del agua en tiempo real. Posteriormente, contando con la información obtenida en tiempo real, teniendo en cuenta la dinámica histórica de la variable de interés $\mathrm{y}$, contando con la dinámica actual e histórica de eventos asociados (naturales como los climáticos, meteorológicos, etc. $y$, antropogénicos como la regulación de volúmenes de agua en los casos pertinentes, etc.) y su propia intuición profesional, el experto de turno pronostica la dinámica futura de la característica en estudio (en nuestro caso el nivel del agua). Si los pronósticos hidrológicos indican una dinámica de la característica en estudio tal, que se prevé un nivel de agua que exige dar un aviso o boletín, entonces el experto de turno emite lo pertinente. Una vez emitido el aviso, boletín o propuesta la alerta, corresponde al usuario tomar las decisiones respectivas" (IDEAM, 2008, p.23). Este procedimiento se ilustra en la figura 3.

Figura 3

Funcionamiento operacional de la toma de decisiones por parte del usuario.

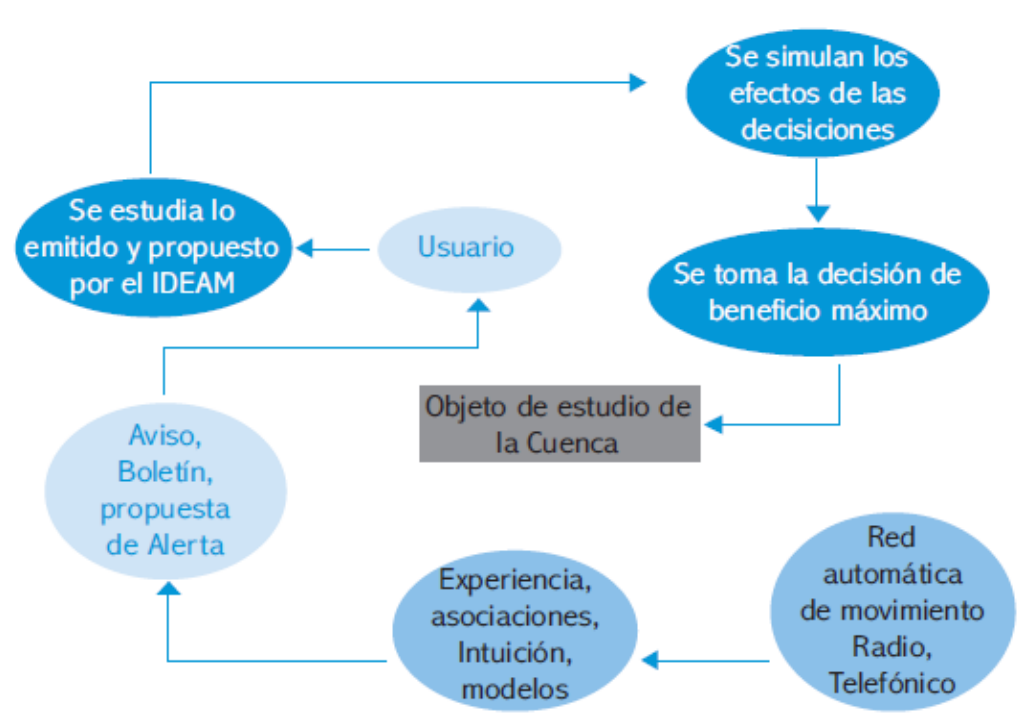

Fuente: IDEAM, (2008). Protocolo para la emisión de los pronósticos hidrológicos. Subdirección de Hidrología. Bogotá.

De acuerdo con el IDEAM (2008), "Como se aprecia en este esquema, el funcionamiento operacional de la toma de decisiones por parte del usuario (ya sea del sector económico, social o ambiental) consiste en lo siguiente: generalmente el usuario obtiene del IDEAM un aviso, un boletín, un comunicado o una propuesta de alerta, luego valiéndose de la dinámica actual e histórica de las medidas a tomar, asociadas al evento probable de suceder y, aplicando su propia intuición y saber profesional, el funcionario responsable en la entidad denominada "usuario" pronostica las consecuencias de la medidas que se tomarían y, posteriormente, de acuerdo a criterios de efectividad u optimización, se toma la decisión para atender la situación de tal manera que se garantice el mejor resultado" (p. 23). 
Como se puede apreciar en las figuras 1 y 2, formalmente la alerta temprana por inundación se emite por parte de un Alcalde o de una autoridad nacional como lo es la Presidencia de la República. Para su emisión exclusivamente se contemplan los aspectos científicos que describen el proceso de formación de una inundación.

Desde el ámbito científico, las previsiones de los eventos de inundación en la cuenca del río Bogotá se realizan con el siguiente procedimiento: a) la autoridad en meteorología e hidrología le corresponde al IDEAM según Decreto 1277 de 1994 y por ello se encarga de realizar a nivel nacional los monitoreos hidro-meteorológicos, así como también emite los pronósticos del estado del tiempo atmosférico y del comportamiento de los ríos, y en los casos necesarios sugiere el tipo de alertas que se podrían emitir; b) una vez se recibe el pronóstico del comportamiento de los ríos de parte del IDEAM, la Corporación Autónoma Regional de Cundinamarca - CAR, entidad pública que cuenta con su propia red hidro-meteorológica en su jurisdicción (con 106 municipios ubicados en los departamentos de Cundinamarca y Boyacá, a excepción del Distrito Capital de Bogotá) detalla los alcances del pronóstico y comunica a las Alcaldías y empresas privadas sobre la amenaza o posibilidad de los eventos de inundación y se disponen las medidas para evitar pérdidas o daños; c) de otra parte, el Distrito Capital de Bogotá, entidad pública que también tiene su propia red hidro-meteorológica, al recibir los avisos del IDEAM y contando con su propia información, dispone de sus recursos (humanos, tecnológicos, etc.) para atender las situaciones de emergencia que puedan ocasionar los eventos de inundación. Está claro que el IDEAM no emite alertas formalmente, pero sí sugiere a las Alcaldías la categoría de éstas que se podrían establecer según la magnitud de los eventos hidro-meteorológicos que se prevén en los pronósticos.

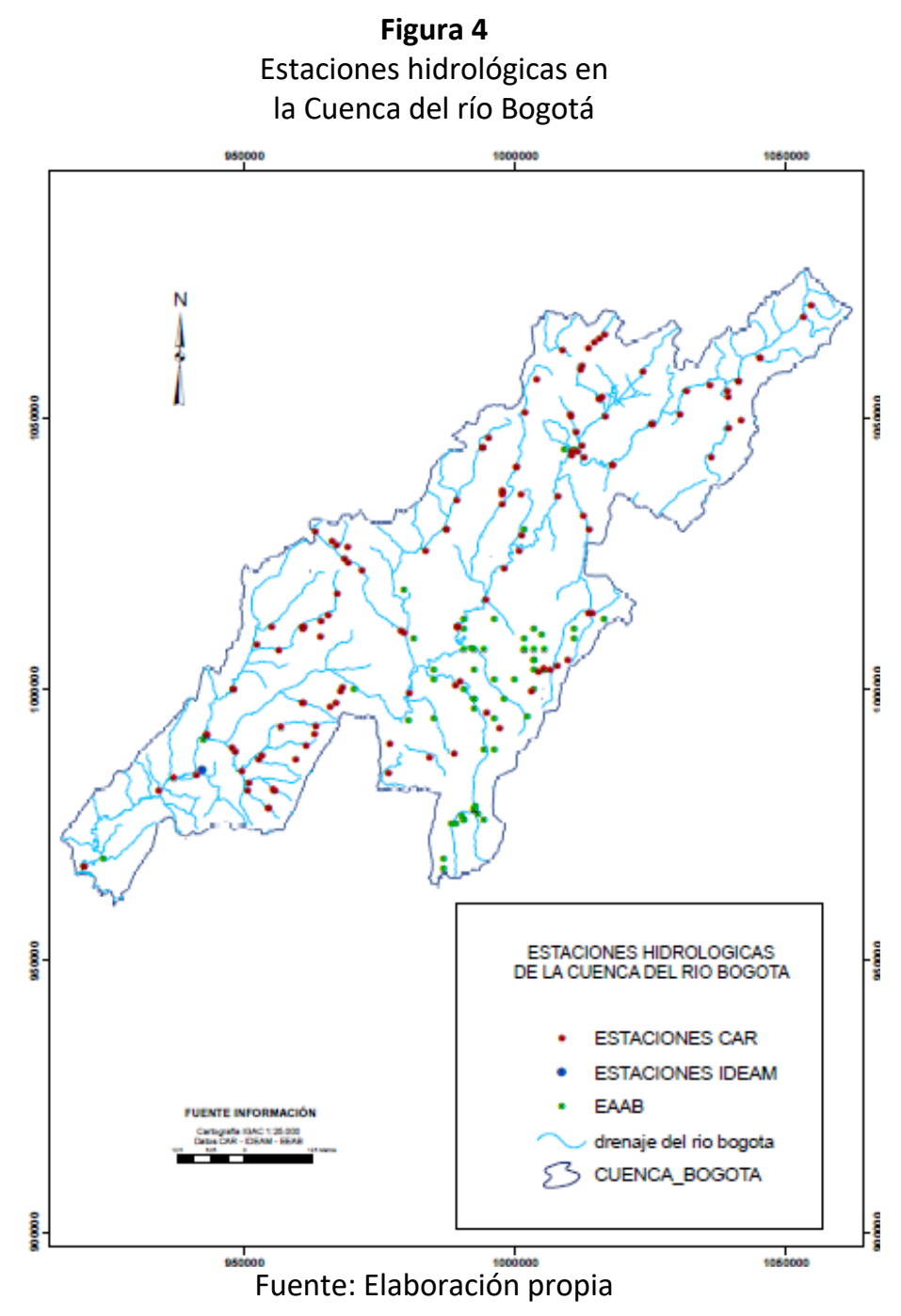




\subsection{El SAT Comunitario en la cuenca del río Bogotá}

Un Sistema de Alerta Temprana Comunitario (SATC) es una abstracción humana que permite desde sus saberes (sociales, culturales, económicos y científicos) poder "ver" o descubrir señales en el acontecer diario (tanto de la actividad humana como de la dinámica natural), saber interpretarlas para "prever" lo que podría suceder a futuro (a corto, mediano y largo plazo) y, ante las previsiones, lograr "decidir" su actuar de tal manera que la afectación se anule o sea en lo posible algo menor. Esta abstracción humana se soporta en conocimientos (populares o científicos), tecnologías (sencillas o complejas) y métodos (tradicionales o modernos) que permiten prever (en el tiempo y espacio) con suficiente tiempo de antelación lo que sucederá en materia de eventos de inundación.

Para la implementación de un SATC en la cuenca del río Bogotá se deben surtir las siguientes etapas: a) diagnóstico de las culturas, religiones que profesan, costumbres y visiones políticas de los gentes y gobernantes que habitan en el espacio geográfico de la cuenca; b) diagnóstico del comportamiento atmosférico e hidrológico de los cuerpos hídricos (artificiales o naturales) en la cuenca; c) optimización del diseño de monitoreo hidrometeorológico en la cuenca; d) implementación de instrumentos (sencillos y modernos) para enriquecer las mediciones en la cuenca; e) identificación de comportamientos o patrones en el mundo faunístico y vegetal que estén correlacionados con el comportamiento atmosférico e hidrológico de los cuerpos hídricos (artificiales o naturales) en la cuenca; f) desarrollo de métodos científicos de pronóstico meteorológico e hidrológico que incluyan los altos niveles de incertidumbre que ofrecen las mediciones; g) inclusión de conocimientos tradicionales o populares de las gentes en los métodos de pronósticos científicos; h) identificación de señales sociales que son aceptadas por las gentes ante situaciones de emergencia; i) identificación de líderes comunitarios en los asentamientos humanos que se encuentran en la cuenca; j) establecimiento de procedimientos científicos y populares para la emisión de alertas por inundación; k) emisión de las alertas por inundación con sentido científico y socialmente aceptables. En la figura 4 se ilustra el esquema general de un SATC para el caso de la cuenca del río Bogotá.

Figura 5

Esquema general de un SATC

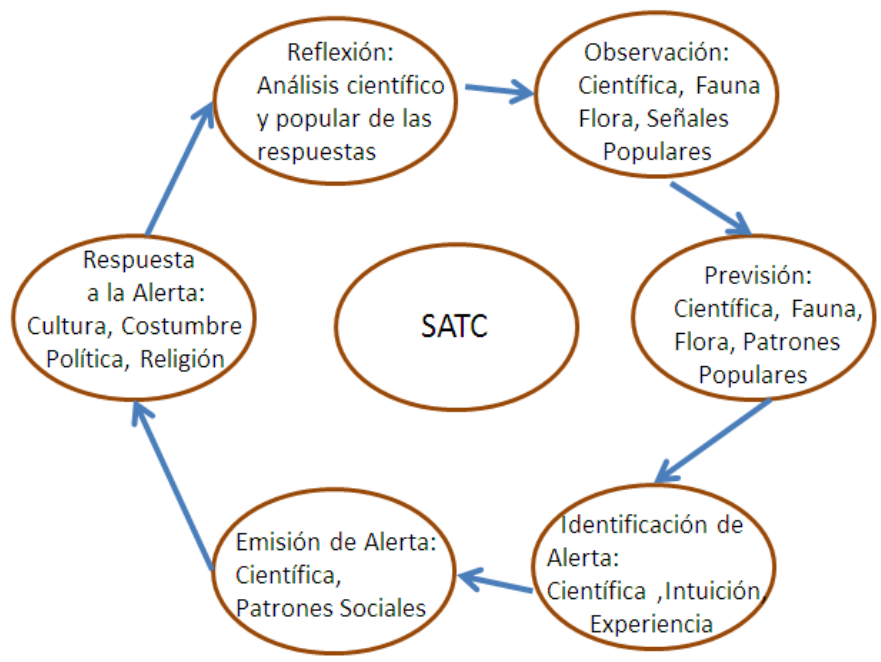

Fuente: Elaboración propia adaptada de los siguientes documentos: OEA, (2010). Manual para el diseño, instalación, operación y mantenimiento de sistemas comunitarios de alerta temprana ante inundaciones e IDEAM,

(2008). Protocolo para la emisión de los pronósticos hidrológicos. Subdirección de Hidrología. Bogotá. 
Al implementar un SATC con la metodología aquí propuesta se está preparando a la sociedad en general para afrontar situaciones de inundación en la cuenca del río Bogotá como una medida de adaptación ante el cambio de sus frecuencias y magnitudes por el fenómeno de cambio climático.

\section{Resultados y discusión}

Respecto a un Sistema de Alerta Temprana Comunitario se pueden establecer dos conclusiones que a primera vista son contrarias: a) la primera se relaciona con el hecho de que la mayoría de ingenieros consideran que el conocimiento popular no es válido, toda vez que carece de la rigurosidad del método científico; b) la segunda que es propia del sentido común y popular considera que la intuición, la costumbre, y la cultura son garantía de que los hechos se pueden prever con sabiduría aunque carezca de rigurosidad metodológica. En el primer caso, es un absurdo contratar a un chamán para garantizar que en un día determinado no llueva, es también incoherente pensar en que la fauna pueda ejercer la actividad de predicción de una inundación o que el famoso "Almanaque Bristol" pueda acertar con las previsiones del clima. En el segundo caso no se pueda negar que las cosas sucedan con cierta mística y que existan leyes divinas y naturales, las cuales con un buen olfato pueden ser adivinadas o previstas.

Es importante resaltar que en ontología se sabe que la ciencia científica está limitada por los cinco sentidos del ser humano y que en general es una ciencia ego-geo-centrista y por lo tanto su capacidad para producir conocimiento tiene un límite. De otra parte, según Aguirre (2004), Ulloa (2011) y Correa (2011) , no se puede despreciar la experiencia acumulada por el ser humano, la fauna y la flora, sino más bien aprovechar esa sabiduría popular y aplicarla en las previsiones de las inundaciones en la cuenca del rio Bogotá. La experiencia de implementar un SATC permitirá corroborar o negar la utilidad de los saberes de las gentes en cada una de sus fases. Esta será una gran oportunidad para ver cómo se enlazan las ciencias exactas, físicas, naturales y las ciencias del espíritu en una ecuación diferencial determinista o estocástica, con la cual se modela un evento de inundación.

\section{Conclusiones}

En Colombia no son muy frecuente las experiencias de los Sistema de Alerta Temprana Comunitario, el cual enlaza los saberes populares y los conocimientos científicos. De esta forma, se puede concluir que el SATC propuesto para la cuenca del río Bogotá será una gran oportunidad para beneficiar a la sociedad que allí se desempeña.

Las fases de un SATC son: a) observación de las variables meteorológicas e hidrológicas y de las señales en el comportamiento humano, en la fauna y en la flora, comparando el comportamiento actual con el previsto por cambio climático; b) la previsión con métodos populares y científicos (modelación de procesos no estacionarios), teniendo en cuenta las señales que arrojan los comportamientos de la flora y fauna; c) la identificación de las situaciones de inundación con orientaciones científicas, experiencias de las gentes y su sentido de intuición; d) emisión de la alerta por inundación con reglas científicas y patrones sociales; e) la respuesta a la alerta expresada en términos de las culturas de las gentes que habitan la cuenca del río, sus costumbres, las visiones políticas y religiosas que comparten; f) reflexión sobre los resultados de la emisión de las alertas en el bienestar de las gentes y sociedad en general, considerando análisis científicos y populares.

Implementar un SATC en la cuenca del río Bogotá es viable, toda vez que sus fases no requieren mayores inversiones económicas, ni tampoco la adquisición de tecnologías costosas. Ello significará vincular profesionales 
de otros ámbitos de las ciencias, muy alejadas de la ingeniería, tales como historiadores, antropólogos, trabajadores sociales, entre otros.

\section{Referencias bibliográficas}

Aguirre, M. R. (2004) Sabidurías campesinas andino - amazónicas. En la madrugada de San Pedro y San Pablo, la presencia de las neblinas es seña para la crianza de las chacras. No 2243.

Banco Interamericano de Desarrollo y Comisión Económica para América Latina y el Caribe, (2012). Valoración de daños y pérdidas Ola invernal en Colombia 2010-2011

Camino, E. R., Ruggeroni, J. R. P., \& Sánchez, F. H. (2015). Quinto informe de evaluación del IPCC: Informe de síntesis. Revista Tiempo y Clima, 5(47).

Correa, S. (2013). Procesos culturales y adaptación al cambio climático: la experiencia en dos islas del Caribe colombiano.

Cruz, K. S., Escobar, Y. C., \& Díaz, Á. J. Á. (2013). Análisis de aspectos que incrementan el riesgo de inundaciones en Colombia. Revista Luna Azul (On Line), (37), 219-238.

Dane (2011), Reporte final de áreas afectadas por inundación 2010 - 2011.

Gonzalez Y, (2004). Sistema de Información Geográfica para Incendios SIGPI. Manual del usuario. Segunda versión. IDEAM, Bogotá.

Garcia, C., Tenorio, A., \& Muñoz, F. (2011). Ciclos naturales, ciclos culturales: Percepción y conocimientos tradicionales de los Nasas frente al cambio climático en Toribio, Cauca, Colombia. Perspectivas culturales del clima. Ed. UN de Colombia. (Bogotá DC), 247-274.

IDEAM, (2008), Protocolo para la emisión de los pronósticos hidrológicos. Subdirección de Hidrología. Bogotá.

IDEAM (2007) Protocolo para el monitoreo y seguimiento del agua. Subdirección de Hidrología. Bogotá.

IDEAM, DELTARES y UNESCO, (2012). Proyecto para la gestión integral del riesgo en los componentes de pronóstico hidrológico y alerta temprana en la cuenca del río Bogotá - perfil del proyecto

IDEAM,(2010). 2a Comunicación Nacional ante la Convención Marco de las Naciones Unidas sobre Cambio Climático: República de Colombia, ISBN: 9789588067315.

Instituto Latinoamericano para una Sociedad y un Derecho Alternativos ILSA, Universidad Nacional de Colombia, ULLOA, A., Editor. (2011). Perspectivas culturales del clima.

NUMA- UFPA, Aldunce, P, Bezanilla, A y Carvajal, Y. (2008). Hacia la Evaluación de Prácticas de Adaptación Ante la variabilidad y el Cambio Climático.

Universidad Externado de Colombia, (2003). Proyecto regional de población, Centro Latinoamericano Y Caribeño de Demografía (CELADE), División de Población de la CEPAL/Fondo de Población de las Naciones Unidas (UNFPA) Población y desarrollo. Santiago de Chile 
Universidad Externado de Colombia, (2007). Ciudad, espacio y población: el proceso de urbanización en Colombia.

ONU, (2009). Terminología sobre Reducción del Riesgo de de Desastres. Estrategia Internacional para la Reducción de Desastres

OEA, (2010). Manual para el diseño, instalación, operación y mantenimiento de sistemas comunitarios de alerta temprana ante inundaciones

Pabón, J. D. (2011). El cambio climático en el territorio de la Corporación Autónoma Regional de Cundinamarca. Pabón Caicedo, J. D. (2011).

Rada, O. P., Zaballa, M. E., Pinto, M. R., Apaza, E. G., \& Mantilla, R. (2009). Segunda Comunicación Nacional del Estado Plurinacional de Bolivia ante la Convención Marco de las Naciones Unidas sobre el Cambio Climático. La Paz: Ministerio de Medio Ambiente y Agua Viceministerio de Medio Ambiente, Biodiversidad y Cambios Climáticos Programa Nacional de Cambios Climáticos.

República de Colombia. Ministerio del Medio Ambiente. Instituto de Hidrología, Meteorología y Estudios Ambientales (IDEAM), coord. Segunda Comunicación Nacional ante la Convención Marco de las Naciones Unidas sobre Cambio Climático.

Ulloa, A. (2011). Políticas globales del cambio climático: nuevas geopolíticas del conocimiento y sus efectos en territorios indígenas. En Ulloa, A., Editor, ILSA.

Universidad Externado de Colombia, (2007. Ciudad, espacio y población: El proceso de Urbanización en Colombia. Centro de Investigación sobre Dinámica Social, Bogotá, agosto 2007. ISBN 978-958-985663-0-3

WMO, (1992). Vocabulario Meteorológico Mundial. OMM

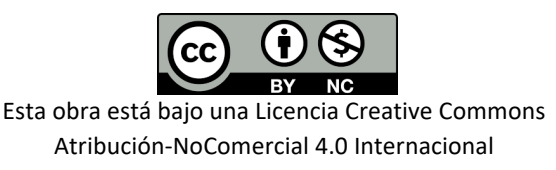

